\title{
The pathologists role in identifying drug toxicity
}

\section{Editorial}

The specialty of pathology has as much complexity in methods and specialization as the radiology specialties. However, pathology initially undertook the clinical laboratory only because it has always been a specialty not involving direct patient contact, except for transfusion medicine, which has both pathology and hematology direction. However, the discipline became more complex as a result of hematological, infectious disease and immunological disorders, and the emergence of automated chemistries. This resulted in segmentation of the laboratory disciplines. While the laboratory divisions were developing, the need for a laboratory information system became clear, and the earliest medical laboratory computers were developed. In the meantime, anatomical pathology evolved into organ specific subspecialties, which further led to a separation of anatomic and clinical pathology pursuits, having different advanced skill requirements. Consequently, the laboratories had $\mathrm{PhD}$ and $\mathrm{MD}$, $\mathrm{PhD}$ skilled doctorates attending to their functions and automation. However, in the last forty years there emerged a difference in complexity between community hospital laboratories and reference laboratories based on volume and complexity of the tests performed. As hospitals have consolidated, the clinical laboratory work either was sent to a reference laboratory or to laboratories within the consolidated network. How this proceeds with a reduced $\mathrm{PhD}$ workforce is to an extent a reimbursement phenomenon.

In addition to this consideration, the need for advanced pharmacological and toxicity monitoring has grown due to the long term development and introduction of new drugs for infectious disease, hematologic disorders, immune diseases, and cancers. The laboratory immediately had a role with the development of measures of immunology, infectious disease, chemistry and surgical pathology. The importance of more specific monitoring of the effects of drugs became necessary, both in the development stage and the introduction phase. The impact of drugs is most prevalent in hepatic disorders, but is also important with respect to immune reactions, renal function and pulmonary function. This has resulted in measurement of drugs in the clinical laboratory. The measurement of the effects of drug toxicity is partly covered by the multitest panel that measures liver enzymes, serum proteins, electrolytes, creatinine and BUN.

Liver toxicity is first among the reasons for the withdrawal from the market of approved drugs during the last decade. A guideline, introduces a stepwise approach in detecting hepatotoxicity signals and conducting studies to identify non-clinical hepatotoxicity. This requires the evaluation of data obtained in non-clinical studies prior to introduction of the drug. Non-clinical studies are designed based on the mechanism of drug action and adverse effects. Key elements in the evaluation of hepatotoxic signals are stated:

a. Identify drug-related effects in clinical pathology parameters in relevant species and determine the magnitude of that effect.

b. Attention should be focused on the comparison of individual animal data prior to the treatment and at different experimental time points rather than using the group mean data for such
Volume 6 Issue I - 2018

\author{
Larry H Bernstein \\ Triplex Medical Diagnostics, USA
}

Correspondence: Larry H Bernstein, Triplex Medical Diagnostics, 54 Firethorn Lane Northampton, MA 0I060, USA Email larry.bernstein@gmail.com

Received: February 22, 2018 | Published: February 27, 2018

comparisons. Hepatotoxicity signals observed in non-rodent studies should be thoroughly investigated.

c. The use of data obtained from mechanistic in vitro and/or in vivo models relies on the collection of hepatotoxic signals as a continuous process covering all phases. Improved detection and prediction of drug-induced hepatotoxicity could be achieved by the use of new predictive biomarkers and in vitro and/or in vivo models, not yet available and/or validated.

When the kidney is exposed to harmful toxics, the damaged kidney is unable to perform the function of excretion of waste. These results in impaired excretion of electrolytes, like magnesium and potassium, and these will be elevated with a metabolic imbalance. ${ }^{2}$ This toxicity occurs from exposure to the halogenated hydrocarbons like carbon tetra chloride, trichloroethylene and the heavy metals like lead and cadmium then toxic injury is caused. There are various types of toxicities that lead to the renal failure by causing acute or chronic injury and subsequently, kidney failure. Therapeutic agents that can adversely affect the kidney result in tubule-interstitial, glomerular or vascular disease, often caused by antibiotics or non-steroidal antiinflammatory drugs. Drug-induced glomerular and vascular disease is relatively rare. Pulmonary drug toxicity is a common and possibly under diagnosed cause of acute and chronic lung disease. ${ }^{3}$ Agents that have potential toxic effects on the lungs include cytotoxic drugs such as bleomycin, methotrexate, and cyclophosphamide and noncytotoxic drugs such as nitrofurantoin, sulfasalazine, and amiodarone. The clinical and radiologic manifestations of pulmonary drug toxicity generally reflect the underlying histopathologic processes. Clinical pathology traditionally comprised clinical chemistry, coagulation, hematology, and urinalysis evaluations as an integral part of the preclinical safety assessment of test studies. ${ }^{4}$ This was expanded with the measurement of drug dose levels. Results of these evaluations provide information regarding the general metabolic processes of organs associated with exposure to tests. These evaluations establish dose-response relationships in preclinical drug safety assessment. The findings are useful for dose selection in chronic studies, and extrapolation to humans for risk assessment and management. The application of clinical pathology testing developing biomarker implementation within the laboratory is also necessary for identifying more sensitive, early signals of drug- induced target organ toxicity. 
The integration of the findings of clinical pathology data with study data is essential in the evaluation of overall safety or risk assessment, but is also relevant for information systems management.

Pathologists play a central role in the recognition and prevention of drug-induced toxicity. Pathologists engaged in anatomic pathology practice must identify a pattern of histological lesions that are interpreted in concert with a variety of clinical data to determine the probable features of drug-induced toxicity and the most likely drug, often of many, to have caused the specific injury. ${ }^{5}$ Toxicological pathologists, working in concert with other scientists, have the responsibility of preventing drug-induced toxicity in humans by identifying potentially toxic drugs. A number of animal species and in vitro and in vivo assays are used in drug development testing. Pathologists integrate study data effectively with traditional morphological evaluation to develop a more detailed grasp of the pathogenesis of drug-induced injury. A number of compounds fail to progress past preclinical development due to limited tools that accurately monitor toxicity in preclinical studies and in the clinic. Research is needed to improve the tools for the detection of organspecific toxicity through the identification and characterization of biomarkers of toxicity. However, there is currently a gap between the scientific work in the development and qualification of novel biomarkers use in drug safety assessment. ${ }^{6}$ The gap is narrowed by identification and application of biomarkers critical for successful drug discovery and development. ${ }^{7}$ Translational safety biomarkers that are minimally invasive and monitor drug-induced toxicity during human clinical trials are essential for assessing toxicities observed in preclinical toxicology studies necessary to determine therapeutic doses. The data obtained during the biomarker qualification phase includes careful consideration of the analytic method used, the biology, pharmacokinetic and pharmacodynamic properties of the biomarker, and the pathophysiology of the process studied.

\section{Acknowledgement}

None.

\section{Conflict of interest}

The authors have no conflict to declare.

\section{References}

1. Non-Clinical Guideline on Drug-Induced Hepatotoxicity, Committee for Medicinal Products for Human Use. European Medicines Agency. London; 2008

2. John R, Herzenberg AM. Renal toxicity of therapeutic drugs. J Clin Path. 2009;62(6):505-556.

3. Rossi SE, Erasmus JJ, McAdams HP, et al. Pulmonary Drug Toxicity: Radiologic and Pathologic Manifestations. Radiographics. 2000;20(5):1245-1259.

4. York MJ. A Comprehensive Guide to Toxicology in Drug Development. 2nd ed. Clinical Pathology. 2017; p. 325-374.

5. Cullen JM, Miller RT. The role of pathology in the identification of drug-induced hepatic toxicity. Expert Opin Drug Metab Toxicol. 2006;2(2):241-247.

6. Campion S, Boekelheide K, Vaidya VS, et al. The current status of biomarkers for predicting toxicity. Expert Opin Drug Metab Toxicol. 2013;9(11):1391-13408.

7. Sasseville VG, Mansfield KG, Brees DJ. Safety Biomarkers in Preclinical Development. Veterinary Pathology. 2013;51(1):281-291. 